\title{
Van Cittert-Zernike theorem for introductory optics course using the concept of fringe visibility
}

Chandrasekhar Roychoudhuri, Kevin Lefebvre

Chandrasekhar Roychoudhuri, Kevin R. Lefebvre, "Van Cittert-Zernike theorem for introductory optics course using the concept of fringe visibility," Proc. SPIE 2525, 1995 International Conference on Education in Optics, (13 October 1995); doi: 10.1117/12.224008

SDIE Event: SPIE's 1995 International Symposium on Optical Science, Engineering, and Instrumentation, 1995, San Diego, CA, United States 


\title{
VAN CITTERT-ZERNIKE THEOREM FOR INTRODUCTORY OPTICS COURSE USING THE CONCEPT OF FRINGE VISIBILITY.
}

\author{
C.S. Roychoudhuri and K.R. Lefebvre
}

Photonics Research Center and Electrical \& Systems Engineering/University of Connecticut/260 Glenbrook Rd, U-157, Storrs, CT 06269/Phone (203) 486-2587, Fax: (203) 486-1033, E-Mail: chandra@eng2.uconn.edu

\section{$\underline{\text { SUMMARY }}$}

Historically, the van Cittert-Zernike (vC-Z) theorem on the far-field degree of coherence due to a finite incoherent source was developed by using complex integral propagating mutual intensity function that is normally beyond the "introductory" mathematical background for many students. In 1920, Michelson demonstrated his steller interferometer to measure the separation between a double-star (two point sources) using Young's double slit on his telescope. He observed that the visibility of the fringes oscillate with the change in the slit separation for a given double-star spacing. Then in 1957, Thompson \& Wolf (TW) carried out an experiment to illustrate the physical meaning of the coherence function as the fringe visibility using Young's double-slit experiment. We propose to use Michelson and TW experiments as the starting point to introduce the $\mathrm{vC}-\mathrm{Z}$ theorem. One can sum the cosine fringes due to each point of the extended incoherent source produced by the double-slit placed at the observation plane where the correlation is to be measured. The visibility of this resultant cosine fringes is precisely the correlation function derived by the customary vC-Z theorem. We use a double Fourier transform lens set up that eliminates the need to develop the rigorous Fraunhofer diffraction integral. One only need to sum the plane waves with regular tilt angles at the lens focal planes. The mathematical formulation becomes easily accessible and the physical meaning of limited increase in the spatial coherence due to the propagation of Huygens-Fresnel wavelets from an incoherent source become physically meaningful. 


\section{INTRODUCTION}

With increasing availability of a wide of variety coherent laser sources extending from infra-red to ultraviolet, the necessity of investing time to understand a conceptually complex theorem by van Cittert and Zernike (vC-Z) on the propagation of partial coherence may be called into question. However, the authors believe it is important for two reasons. First, many optical instruments, particularly high resolution microscopes, use incoherent light as the source of illuminating the target under observation.

A good design of such instruments require a proper understanding of the evolution of partial coherence as the illuminating beam propagates through the instruments. Second, the exercise to understand the physics behind increasing spatial coherence due to expanding Huygens-Fresnel wavelets is very useful in appreciating the following concepts: (i) The position of interference fringes can be precisely identified by the order of interference given by the relative path delay between the interfering beams in number of waves. (ii) The "incoherence" is due to averaging effect of different order fringes produced by different parts of an extended source (spatial or spectral).

The foundation of $\mathrm{vC}-\mathrm{Z}$ theorem is based on the observations that one can produce visible double-slit cosine fringes only if the slits are placed sufficiently far from an extended incoherent source. The size of the original source and its distance from the double-slit dictate the contrast or the visibility of the Young's cosine fringes. For vC- $\mathrm{Z}$ theorem, normally the incoherent source is assumed to have a very narrow spectral width. As the spectral width increases, the domain of high contrast fringes continues to narrow toward the zero-order fringes (where the relative path delay between the signals from the two slits is zero). The normalized degree of coherence (or correlation) between the electromagnetic fields at two points on the far-field plane due to a parallel incoherent source is given by the Fourier transform of the incoherent source function. The running variable on the far-field plane is not the running coordinate(s), but the separation between the two points, whose correlation is being considered. Functionally, it is equivalent to the Fraunhofer (far-field) diffraction pattern due to a coherently illuminated aperture whose functional form is equivalent to the incoherent intensity distribution. 
It is to be noted that the Fraunhofer diffraction pattern due to a coherent source manifests itself as a directly observable intensity variation on the far-field plane itself. However, an incoherent source produces a uniform intensity at the farfield. Even though the abstract optical correlation behavior in this plane is mathematically given by the Fourier transform of the incoherent source function, it remains hidden behind the uniform intensity. The functional correlation behavior is indirectly measurable by the visibility of the Young's cosine fringes by placing a variable-separation double-slit on the plane of observation. [For alternate expression of $\mathrm{vC}-\mathrm{Z}$ theorem, see footnote]

In the next two sections (2 and 3), we will develop the basic tools of Young's double-slit experiment using Huygens-Fresnel's wavelet propagation method along with the quadratic lens transfer function. Section 3 shows the equivalence of correlation and visibility. Section 4 uses these tools to develop the vC-Z theorem for one-dimensional incoherent rectangular slit source. Section 5 illustrates Michelson's Steller Interferometer used to measure the separation of double-stars.

We will graphically show how the far-field correlation or the visibility due to two point sources (stars) change as the two sets of unit-visibility cosine fringes slide over each other with increasing separation between the double-slit. Section 6 builds on section 5 and graphically illustrates how the far-field correlation function various with the increasing distance between the correlating points while the outer source size remains fixed. Section 7 graphically illustrates how the correlation function varies for a fixed pair of points while increasing the source size.

1. Born and Wolf, "Principles of Optics" Pergamon Press: (a) van Cittert-Zernike theorem-Ch.10; (b) Michelson Steller Interferometer - Ch. 7.

2. Saleh and Teich, "Fundamentals of Photonics", Wiley Interscience. Ch.-10.

3. Thompson and Roychoudhuri, Optica Acta $\underline{26}$ (1), 21-34, 1979; “On the propagation of coherent and partially coherent light." 


\section{YOUNG'S DOUBLE SLIT EXPERIMENT I: COMPLETELY COHERENT CASE; POINT SOURCE ILLUMINATION}

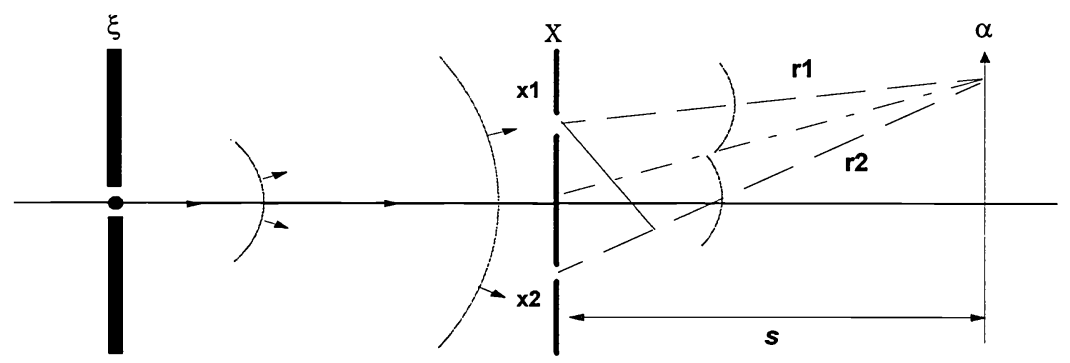

- Interference Equation:

The resultant intensity $\mathrm{I}(\alpha)$ at $\alpha$ is given below due to the superpositon of complex amplitudes $\mathrm{V}\left(\mathrm{x}_{1}\right)$ and $\mathrm{V}\left(\mathrm{x}_{2}\right)$ arriving at $\alpha$ from $\mathrm{x}_{1}$ and $\mathrm{x}_{2}$ experience a delay $\phi_{1}=\mathrm{k}_{1} \mathrm{r}$ and $\phi_{2}=\mathrm{k}_{2} \mathrm{r}$ respectively:

$$
\begin{aligned}
\mathrm{I}(\alpha) & \left.=\left|\mathrm{V}\left(\mathrm{x}_{1}\right)+\mathrm{V}\left(\mathrm{x}_{2}\right)\right|^{2}=\mid V \mathrm{e}_{1}^{i \varphi_{1}}+V_{2} \mathrm{e}^{i \varphi_{2}}\right)\left.\right|^{2} \\
& =\left|V_{1}\right|^{2}+\left|V_{2}\right|^{2}+2 V_{1} V_{2} \cos \left(\varphi_{2}-\varphi_{1}\right)
\end{aligned}
$$

For symmetric slits and illumination the amplitude $\left|V_{2}\right|^{2}+\left|V_{1}\right|^{2}=I_{0}$ and assuming $\phi_{2}-\phi_{1}=\phi_{12}$ then: $\mathrm{I}(\alpha)=2 \mathrm{I}_{\mathrm{o}}\left(1+\cos \varphi_{12}\right)$

- Visibilty (Fringe Contrast):

For a point source at the $\xi$-plane, the illumination at the x-plane is completely coherent and the fringe visibility or contrast is perfect or unity.

$$
\boldsymbol{V} \equiv \frac{I_{\max }-I_{\text {min }}}{I_{\text {max }}+I_{\text {min }}}=1
$$




\section{YOUNG'S DOUBLE SLIT EXPERIMENT II: PARTIALLY COHERENT} CASE; EXTENDED SOURCE ILLUMINATION

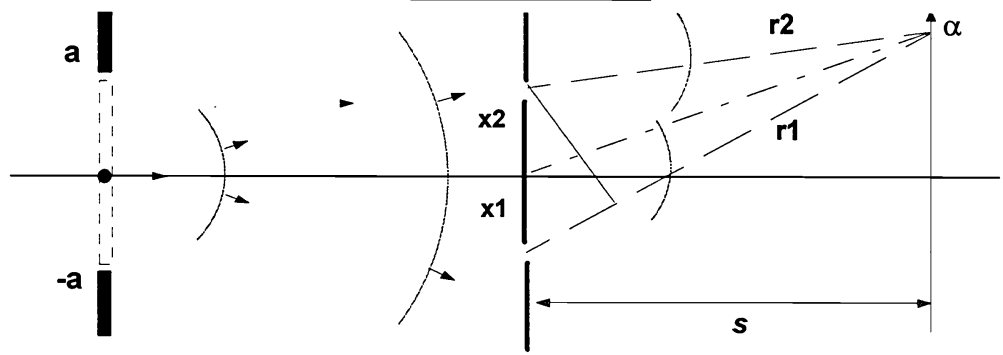

- Interference Equation:

The (ensemble or time) averge intensity, $\mathrm{I}(\alpha)$, at $\alpha$ is due to the superposition of complex amplitudes $\mathrm{V}\left(\mathrm{x}_{1}\right)$ and $\mathrm{V}\left(\mathrm{x}_{2}\right)$ arriving at $\mathrm{x}_{1}$ and $\mathrm{x}_{2}$.

(propagation constant $\mathrm{K}$ is omitted in the following derivation)

$$
\begin{aligned}
\mathrm{I}(\alpha) & =\left\langle|\mathrm{U}(\alpha)|^{2}\right\rangle=\left\langle\left|\mathrm{V}\left(\mathrm{x}_{1}\right)+\mathrm{V}\left(\mathrm{x}_{2}\right)\right|^{2}\right\rangle \\
& =\left\langle\left|\mathrm{V}\left(\mathrm{x}_{1}\right)\right|^{2}\right\rangle+\left\langle\left|\mathrm{V}\left(\mathrm{x}_{2}\right)\right|^{2}\right\rangle+2 \operatorname{Re}\left\langle\mathrm{V}^{*}\left(\mathrm{x}_{1}\right) \mathrm{V}\left(\mathrm{x}_{2}\right)\right\rangle \\
& =\mathrm{I}_{1}(\alpha)+\mathrm{I}_{2}(\alpha)+2\left|\mathrm{G}_{12}\right| \cos \varphi_{12} \\
& =\mathrm{I}_{1}+\mathrm{I}_{2}+2 \sqrt{\mathrm{I}_{1} \mathrm{I}_{2}} \mathrm{~g}_{12} \cos \varphi_{12}, \text { where } \mathrm{g}_{12} \equiv \frac{\left\langle\mathrm{V}_{1}^{*} \mathrm{~V}_{2}\right\rangle}{2 \sqrt{\mathrm{I}_{1} \mathrm{I}_{2}}} \\
& =\left(1+\beta \mathrm{g}_{12} \cos \varphi_{12}\right)\left(\mathrm{I}_{1}+\mathrm{I}_{2}\right), \text { where } \beta \equiv \frac{2 \sqrt{\mathrm{I}_{1} \mathrm{I}_{2}}}{\left(\mathrm{I}_{1}+\mathrm{I}_{2}\right)}
\end{aligned}
$$

The cosine term represents the optical interference.

- Visibility (Fringe Contrast):

A measure of the strength or the contrast of the interference pattern by Michelson.

$$
\mathcal{V}=\frac{I_{\text {max }}-I_{\text {min }}}{I_{\text {max }}+I_{\text {min }}}=\frac{2 \sqrt{I_{1} I_{2}} g_{12}}{I_{1}+I_{2}}=\beta g_{12} \text {. }
$$

Where $\mathcal{V} \mathrm{g}_{12}$ when $\mathrm{I}_{1}=\mathrm{I}_{2}=\mathrm{I}_{\mathrm{o}}$, leading $\beta=1$. 


\section{TOOLS FOR van CITTERT-ZERNIKDE THEOREM}

The zero-order fringe position on the $\alpha$-plane shifts with the source poistion at the $\xi$-plane. The optical setup corresponds to the double Fourier transform to simulate the van Cittert-Zernike far-field and Young's double slit in the far field. Where $\xi$ and $\alpha$ are conjugate image planes of each other.

- $\quad$ Source on $z$-axis

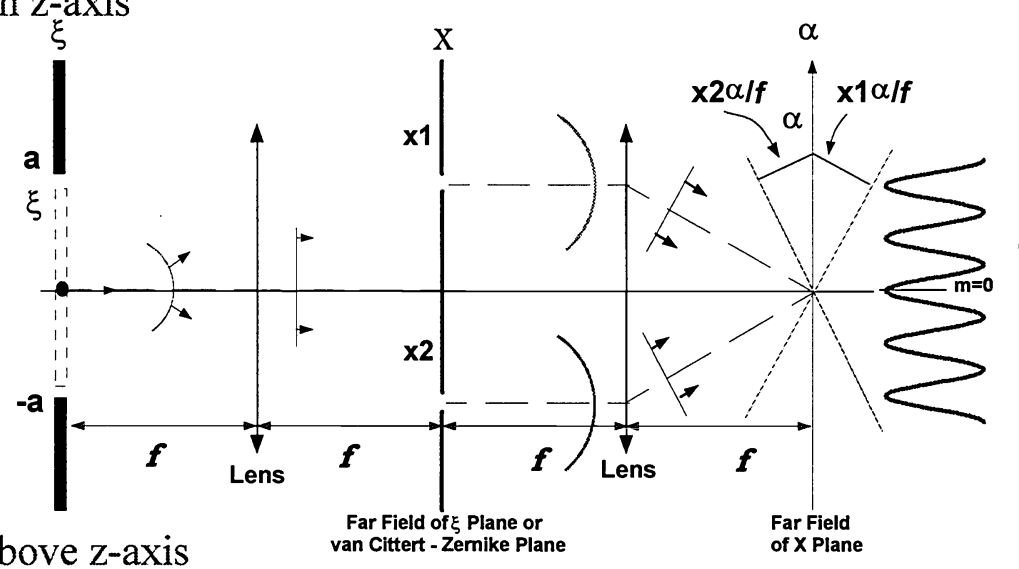

- $\quad$ Source above z-axis

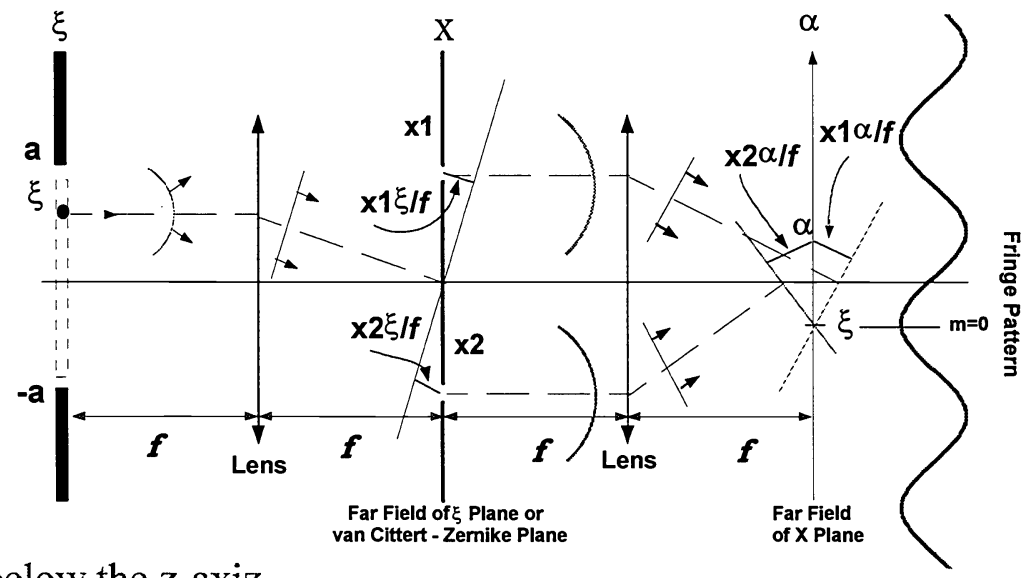

- Source belows the z-axiz

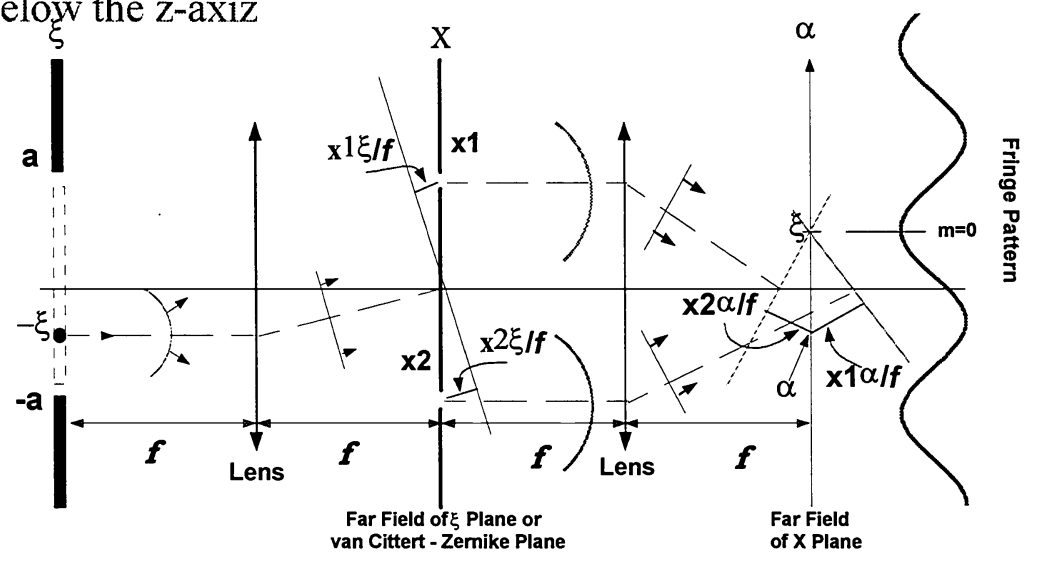




\subsection{Derivation for van Cittert - Zernike Theorem}

Assume the source is divided into elenments $\mathrm{d} \xi_{1}, \mathrm{~d} \xi_{2}, \ldots$ etc. at $\xi_{1}, \xi_{2}, \ldots$ etc. If $\mathrm{V}\left(\mathrm{x}_{1}\right)$ and $\mathrm{V}\left(\mathrm{x}_{2}\right)$ are elemental complex amplitudes at $\mathrm{x}_{1}$ and $\mathrm{x}_{2}$ due to the element $\mathrm{d} \xi_{\mathrm{m}}$, then the total disturbances, neglecting the propagation constant, are:

$$
\begin{array}{lll}
\mathrm{V}\left(\mathrm{x}_{1}\right)=\sum_{\mathrm{m}} \mathrm{V}_{\mathrm{m} 1}\left(\mathrm{x}_{1}\right) \quad \text { and } & \mathrm{V}\left(\mathrm{x}_{2}\right)=\sum_{\mathrm{m}} \mathrm{V}_{\mathrm{m} 2}\left(\mathrm{x}_{2}\right) \\
\mathrm{V}\left(\mathrm{x}_{1}\right)=\sum_{\mathrm{m}} \mathrm{U}\left(\xi_{\mathrm{m}}\right) \exp \left(-i \mathrm{k} \xi_{\mathrm{m}} \mathrm{x}_{1} / f\right) & \mathrm{V}\left(\mathrm{x}_{2}\right)=\sum_{\mathrm{m}} \mathrm{U}\left(\xi_{\mathrm{m}}\right) \exp \left(-i \mathrm{k} \xi_{\mathrm{m}} \mathrm{x}_{2} / f\right)
\end{array}
$$

where $\xi_{\mathrm{m}} \mathrm{x}_{1} / f$ and $\xi_{\mathrm{m}} \mathrm{x}_{2} / f$ are the relative phase delays at $\mathrm{x}_{1}$ and $\mathrm{x}_{2}$ for the tilted plane wave at the $x$ - plane due to the Huygens-Fresnel spherical wavelet originating at $\xi_{\mathrm{m}}$.

Then the correlation function becomes:

$$
\begin{aligned}
\mathrm{G}\left(\mathrm{x}_{1}, \mathrm{x}_{2}\right) & =\left\langle\mathrm{V}^{*}\left(\mathrm{x}_{1}\right) \mathrm{V}\left(\mathrm{x}_{2}\right)\right\rangle \\
& =<\sum_{\mathrm{m}}\left\{\mathrm{V}_{\mathrm{m} 1}\left(\mathrm{x}_{1}\right)\right\}^{*} \sum_{\mathrm{m}} \mathrm{V}_{\mathrm{m} 2}\left(\mathrm{x}_{2}\right)> \\
& =\sum_{\mathrm{m}}<\left\{\mathrm{V}_{\mathrm{m} 1}\left(\mathrm{x}_{1}\right)\right\}^{*} \mathrm{~V}_{\mathrm{m} 2}\left(\mathrm{x}_{2}\right)>+\sum_{\mathrm{m} \neq \mathrm{n}} \sum<\left\{\mathrm{V}_{\mathrm{m} 1}\left(\mathrm{x}_{1}\right)\right\}^{*} \mathrm{~V}_{\mathrm{n} 2}\left(\mathrm{x}_{2}\right)>
\end{aligned}
$$

For incoherent source points, when $\mathrm{m} \neq \mathrm{n}$, the correlation is zero, or

$$
\begin{aligned}
\mathrm{G}\left(\mathrm{x}_{1}, \mathrm{x}_{2}\right) & =\sum_{\mathrm{m}}<\left\{\mathrm{V}_{\mathrm{m} 1}\left(\mathrm{x}_{1}\right)\right\}^{*} \mathrm{~V}_{\mathrm{m} 2}\left(\mathrm{x}_{2}\right)> \\
& =\sum_{\mathrm{m}}<\mathrm{U}^{*}\left(\xi_{\mathrm{m}}\right) \exp \left(i \mathrm{k} \xi_{\mathrm{m}} \mathrm{x}_{1} / f\right) \mathrm{U}\left(\xi_{\mathrm{m}}\right) \exp \left(-i \mathrm{k} \xi_{\mathrm{m}} \mathrm{x}_{2} / f\right)> \\
& =\sum_{\mathrm{m}}<\mathrm{U}^{*}\left(\xi_{\mathrm{m}}\right) \mathrm{U}\left(\xi_{\mathrm{m}}\right)>\exp \left(i \mathrm{k} \xi_{\mathrm{m}}\left(\mathrm{x}_{1}-\mathrm{x}_{2}\right) / f\right) \\
& =\sum_{\mathrm{m}} \mathrm{I}\left(\xi_{\mathrm{m}}\right) \exp \left(i \mathrm{k} \xi_{\mathrm{m}}\left(\mathrm{x}_{1}-\mathrm{x}_{2}\right) / f\right) .
\end{aligned}
$$

For a continuous source we replace the summation by an integral,

$$
\mathrm{G}\left(\mathrm{x}_{1}, \mathrm{x}_{2}\right)=\int \mathrm{I}\left(\xi_{\mathrm{m}}\right) \exp \left(i \mathrm{k} \xi_{\mathrm{m}}\left(\mathrm{x}_{1}-\mathrm{x}_{2}\right) / f\right) \mathrm{d} \xi .
$$

This is the Fourier Transform of the intensity function and is functionally simular to the Fraunhofer diffraction pattern. For a one-dimensional rectangular source of uniform intensity $\mathrm{A}$ and of width $2 \mathrm{a}$, the correlation function is integrated over the source yielding:

$$
\mathrm{G}\left(\mathrm{x}_{1}, \mathrm{x}_{2}\right)=2 \mathrm{Aa} \operatorname{sinc}\left[\mathrm{ka}\left(\mathrm{x}_{2}-\mathrm{x}_{1}\right) / f\right] \text {. }
$$




\section{MICHELSON STELLAR INTERFEROMETER FOR A DOUBLE STAR}

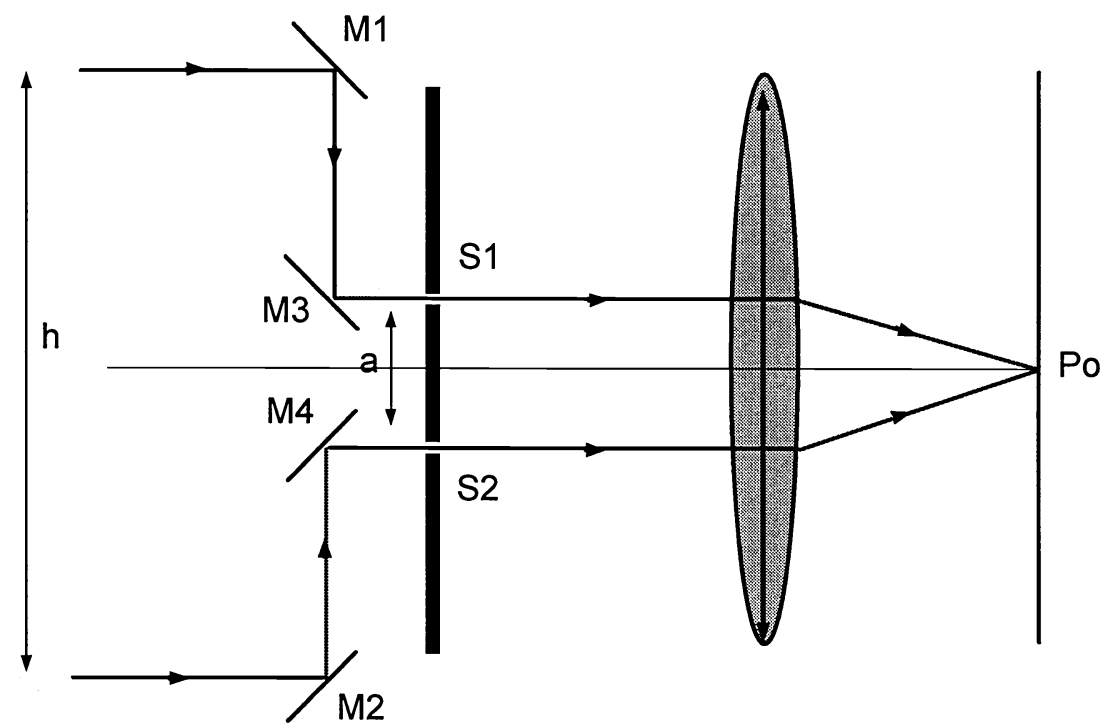

Michelson's original setup for measuring the separation between any double star.

To regular terrestrial telescopes most of the stars, except red giants, appear to be point sources. So a double star is equivalent to two point sources with a finite spacing. Then the double slit in Michelson's Stellar Interferometer is equivalent to Young's double slit experiment with two spatially separate point sources. Each point source creates its own coherent wavefront on the double slit and corresponding cosine fringes, except the zero order fringe position is shifted to the corresponding image position. As the slit separation changes, the summation of the two incoherent cosine fringe systems gives rise to an oscillating fringe visibility as demonstrated in the next section. 


\section{1 OSCILLATIONS OF VISIBILITY DUE TO A DOUBLE STAR A Graphical Presentation}

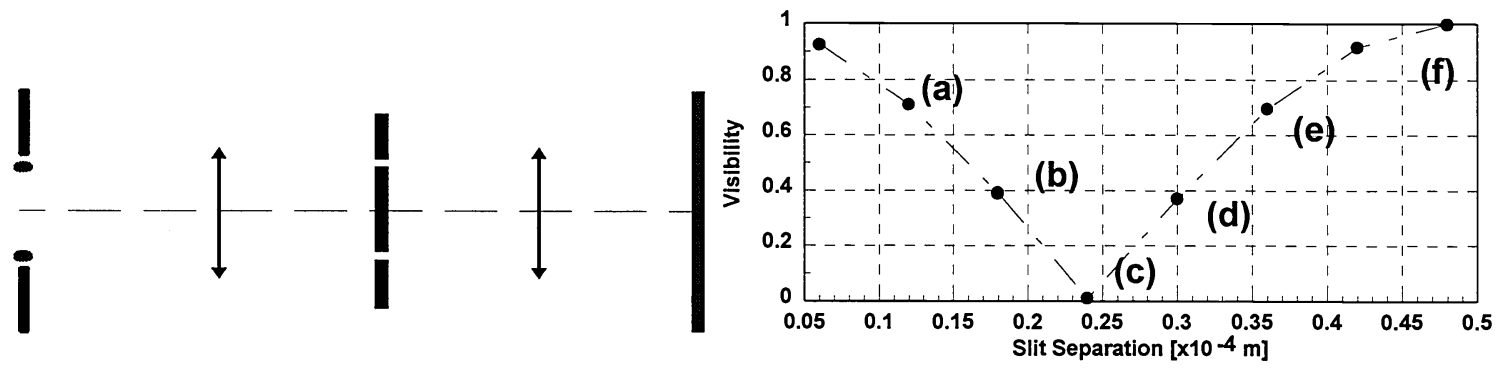

Individual intensities and normalized total intensities for: Two point sources $600 \mu \mathrm{m}$ apart; Focal Length $=.1 \mathrm{~m}$ and $\lambda=.58 \mu \mathrm{m}$.
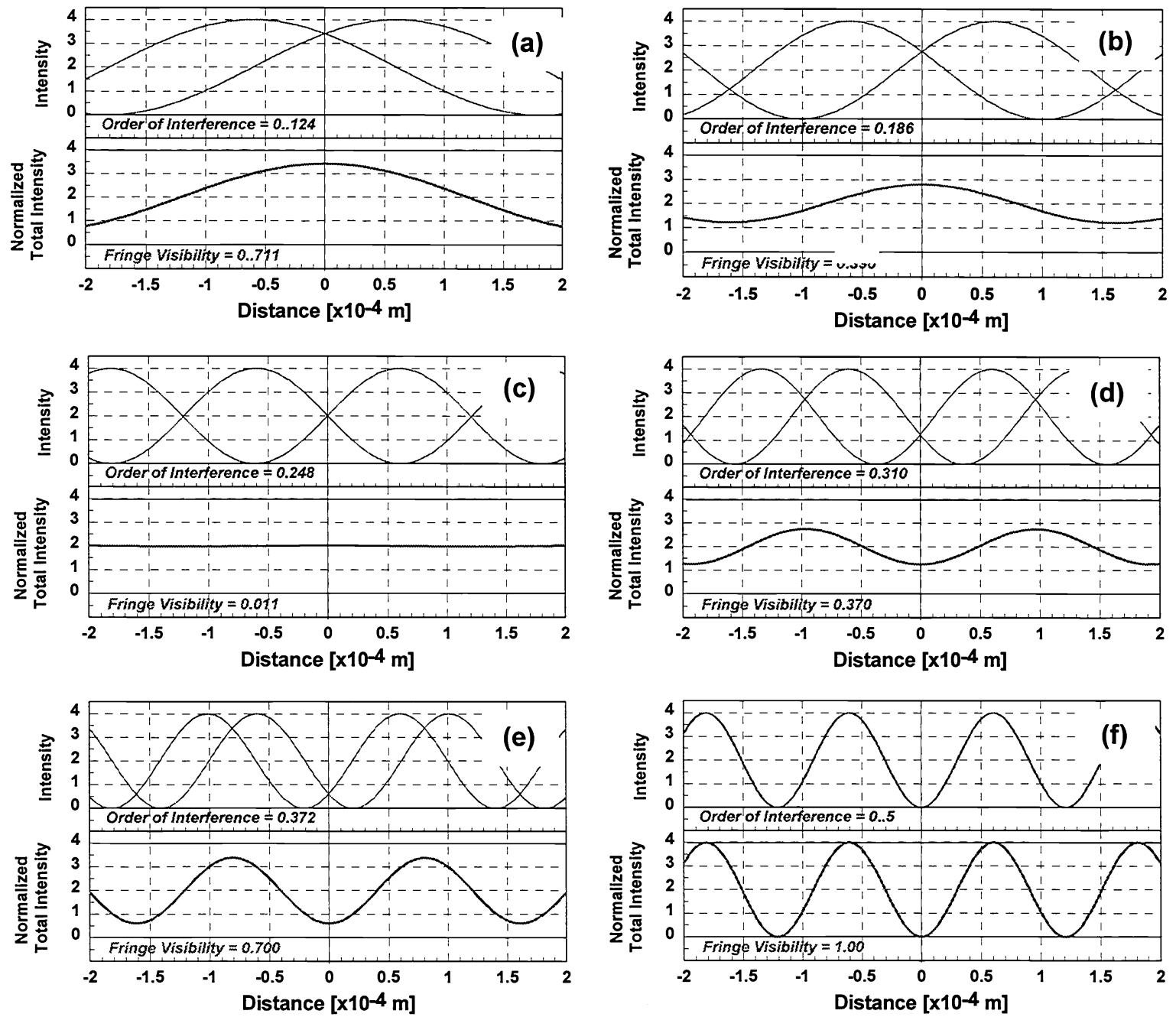


\section{VARIATION OF CORRELATION OR VISIBILITY BETWEEN TWO POINTS OF INCREASING SEPARATION FOR THREE EXTENDED SOURCE.}

\subsection{A Graphical Presentation For Three Point Sources}
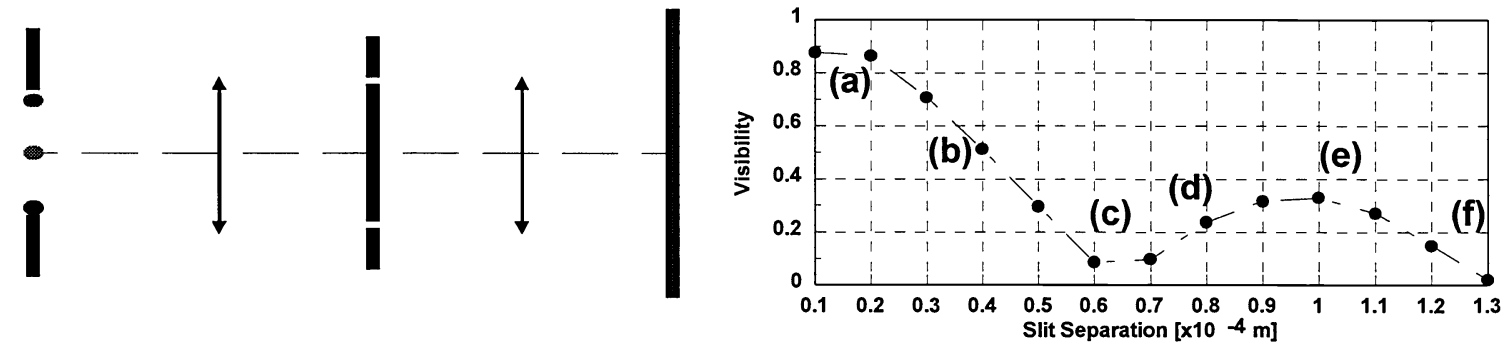

Individual intensities and normalized total intensities for: Three point sources $300 \mu \mathrm{m}$ apart; Focal Length $=.1 \mathrm{~m}$ and $\lambda=.58 \mu \mathrm{m}$.
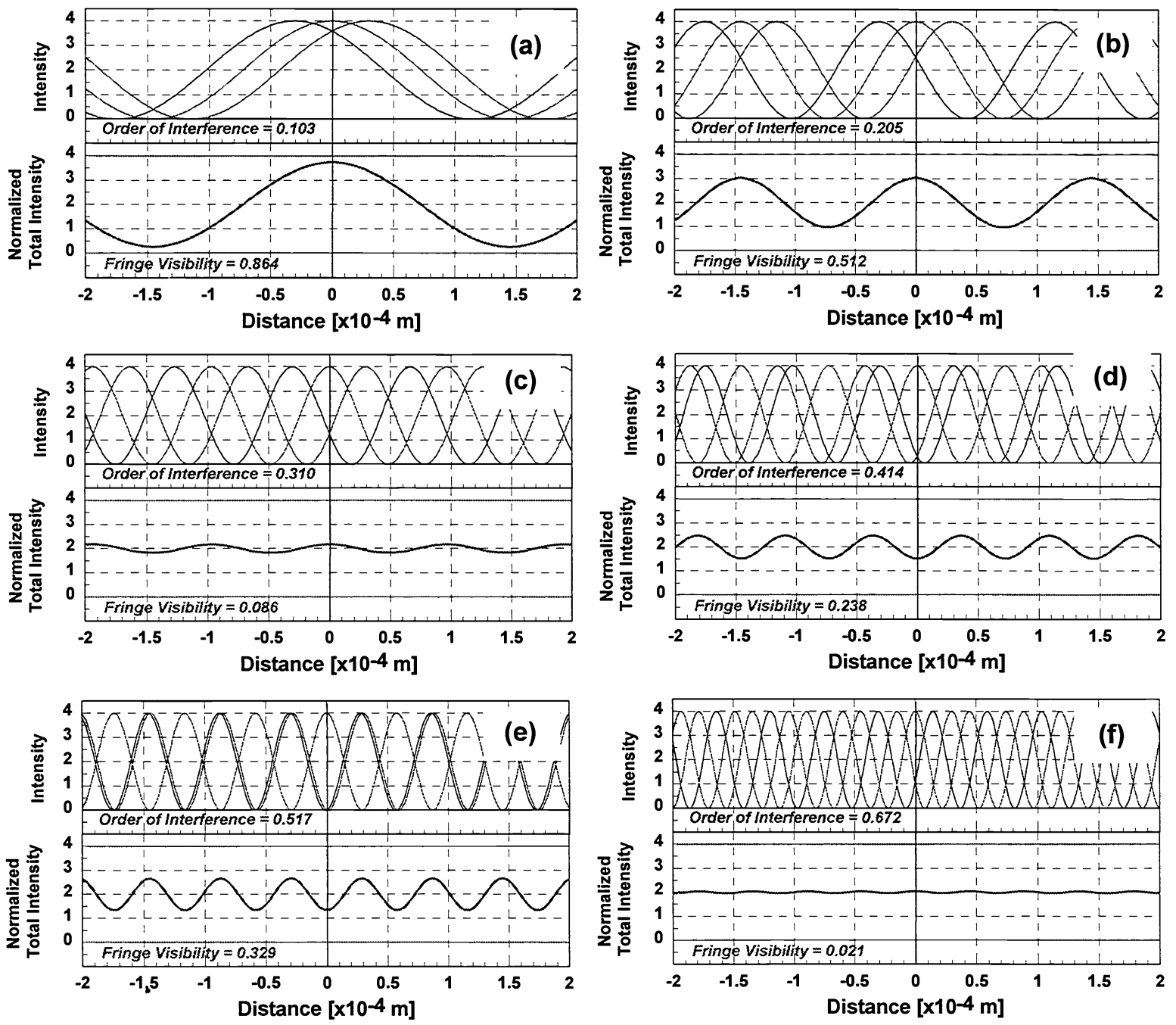


\subsection{A Graphical Presentation For Three Point Sources}

In the limit, the correlation function becomes a sinc function which is the Fourier transform of a rectangular source.

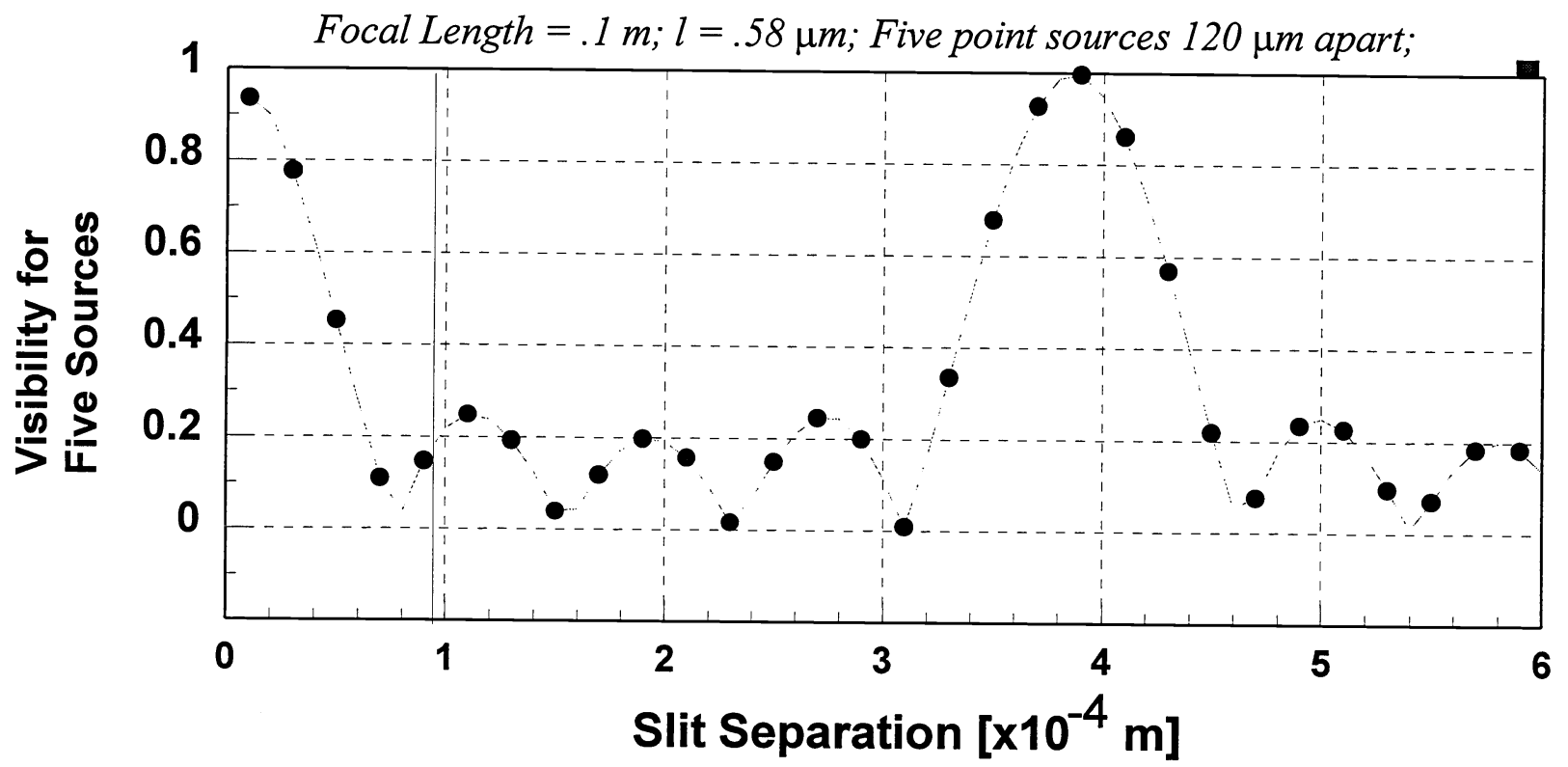

Focal Length $=.1 \mathrm{~m} ; l=.58 \mu \mathrm{m} ;$ Fifteen point sources $40 \mu \mathrm{m}$ apart;

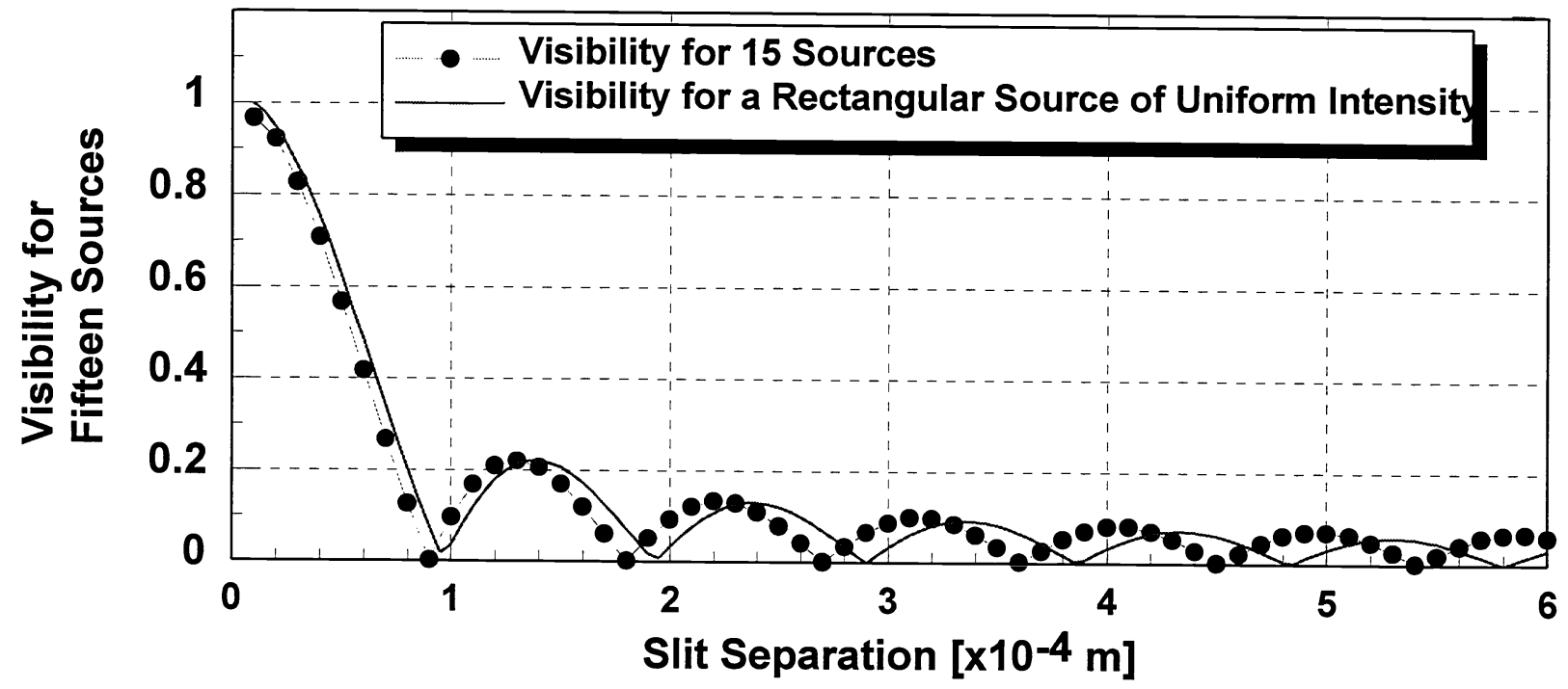




\section{VARIATION OF CORRELATION OR VISIBILITY FOR A FIXED PAIR OF POINTS AS THE SOURCE SIZE INCREASES.}

\subsection{A Graphical Presentation With Increasing Number of Point Sources}

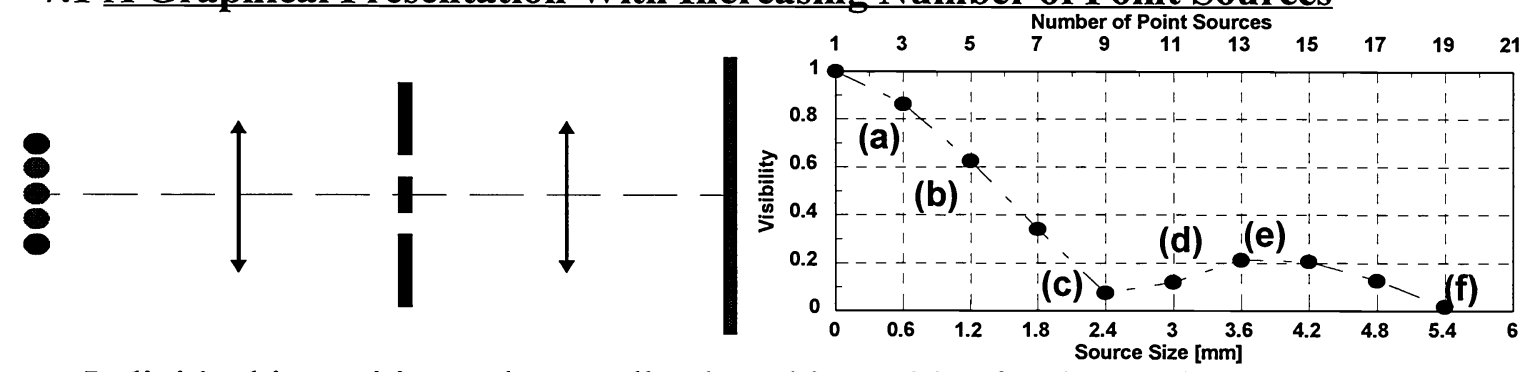

Individual intensities and normalized total intensities for: increasing number of point sources $300 \mu \mathrm{m}$ apart; focal length $=.1 \mathrm{~m}$ and $\lambda=.58 \mu \mathrm{m}$.
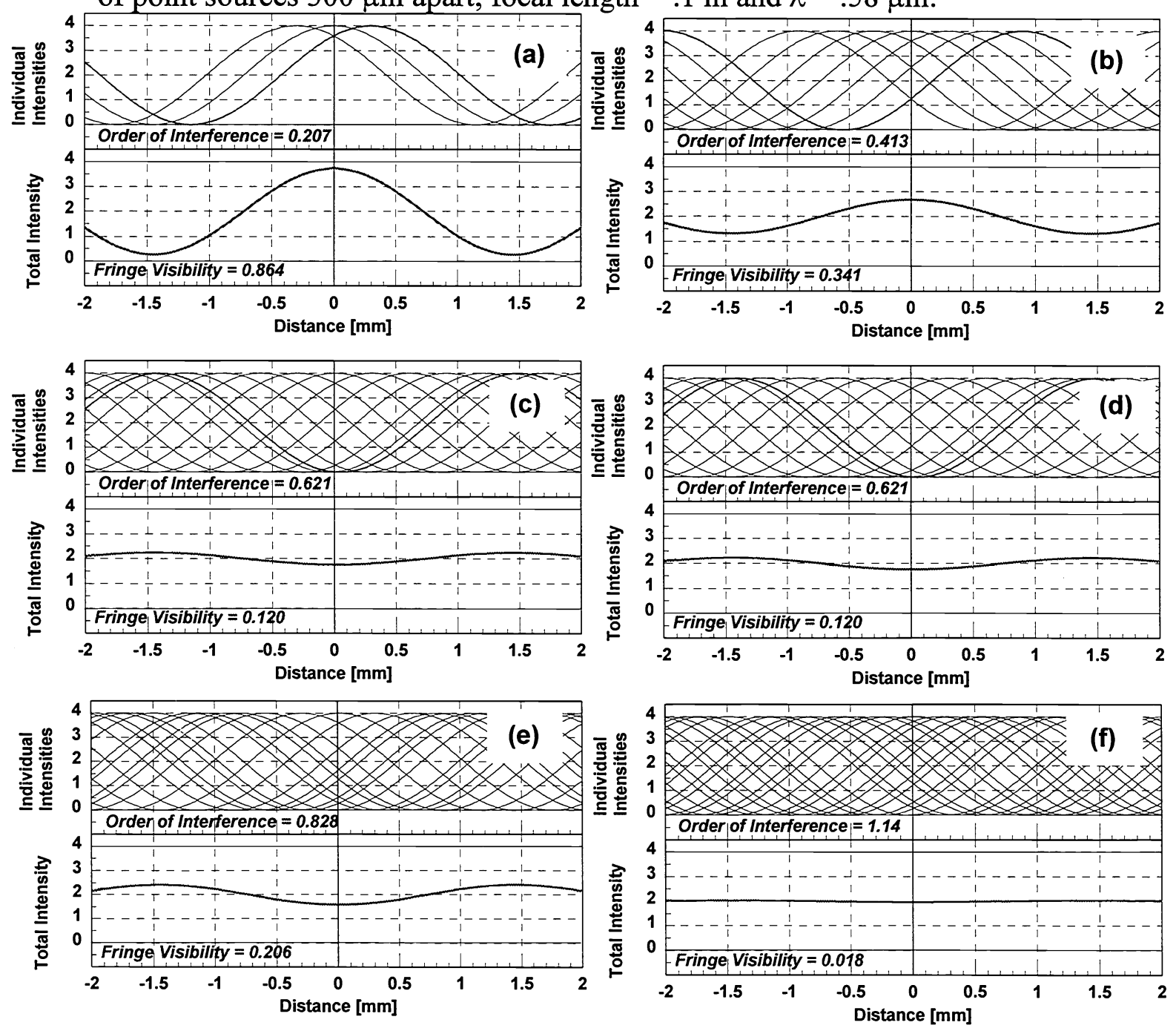
7.2 Visibility for an Increasing Number of Point Sources for Two Slit Spacings

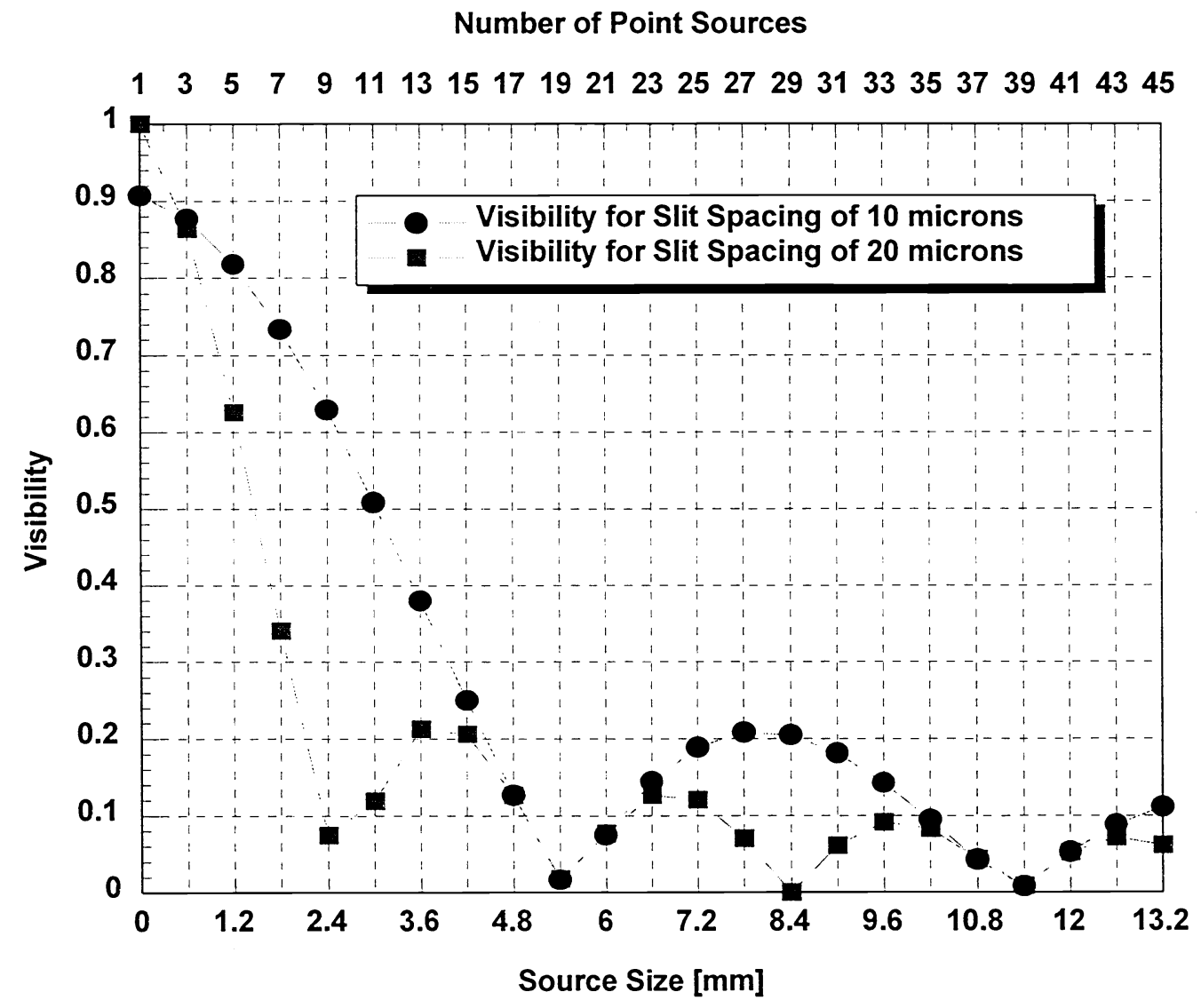

\section{ACKNOWLEDGEMENT}

The authors gratefully acknowledge partial support for this work by the Connecticut Innovation Incorporated through the Critical Technologies Grant and by NSF through the program National Alliance for Photonics Education in Manufacturing or NAPEM (Program\# ELS 94 14669). 